Reprod. Nutr. Dévelop., 1987, 27 (3), 649-657.

\title{
Digestion des protéines de lait et métabolisme de la méthionine chez l'agneau nouveau-né
}

\author{
P. PATUREAU-MIRAND, C. CHAMPREDON, J. PRUGNAUD
}

avec la collaboration technique de O. BERNARD, M. SALLAS, A. SELLE et M. C. VALLUY

Laboratoire d'Etudes du Métabolisme azoté

I.N.R.A. Theix 63122 Ceyrat, France

Summary. Protein digestion and methionine metabolism in newborn lambs.

Methionine absorption and catabolism were studied in 4 newborn lambs during the first $8 \mathrm{~h}$ after birth. Lambs were hourly fed $50 \mathrm{ml}$ goat milk labelled with ${ }^{35} \mathrm{~S}$-methionine and ${ }^{35} \mathrm{~S}$-cysteine. The free amino acid levels and the specific activity of free methionine were measured in jugular blood samples collected $1 \mathrm{~h}$ (just before the first meal), 4, 6 and $8 \mathrm{~h}$ after birth and in the portal blood $8 \mathrm{~h}$ after birth. Specific activities of protein-bound methionine and cysteine were measured in the milk and then in the abomasal and intestinal contents as well as in the liver, intestine and whole body proteins, $8 \mathrm{~h}$ after birth.

The jugular blood levels of free valine, isoleucine, leucine, phenylalanine and histidine increased significantly between 1 and $8 \mathrm{~h}$ whereas the levels of free alanine, serine, glycine, citrulline and 3-methylhistidine decreased. The concentrations of most free amino acids were $30 \%$ higher in portal than in jugular blood. In the abomasal contents, the specific activities of methionine and cysteine were 96 and $168 \%$, respectively of that of ingested milk and in the intestinal contents the corresponding values were 24 and $31 \%$ (table 1). In the jugular blood, the specific activity of methionine reached a plateau before $5 \mathrm{~h}$ after the first meal ; in the portal blood $8 \mathrm{~h}$ after birth it represented $75 \%$ of the specific activity entering the small intestine. The blood methionine flux was calculated according to two methods : 1) from whole-body protein synthesis rates and methionine catabolism and 2) from the irreversible loss of blood methionine (table 2). Comparison of these estimates shows that in newborn lambs fed on goat milk, nearly all methionine entering the intestine may be available at the peripheral blood level. However, a portion of the absorbed labelled methionine did not seem to appear in the portal blood as free methionine. Methionine catabolism differed widely between the four lambs (table 2) and this variation was related to that of the specific activity of cysteine in the liver proteins (fig. 1).

\section{Introduction.}

Chez les nouveau-nés, la digestion des protéines alimentaires est particulière, tout spécialement dans les premières heures qui suivent la naissance chez les ruminants. En effet, la détermination des activités des principales enzymes protéolytiques du tractus digestif a montré que ce potentiel hydrolytique est relative- 
ment faible à cet âge ; seule l'activité de la chymosine abomasale, qui provoque la coagulation de la caséine du colostrum, est élevée, chez le veau comme chez l'agneau, alors que celle des principales protéases pancréatiques (chymotrypsine, trypsine) est basse (Guilloteau et al., 1985). De plus, l'intestin grêle présente une perméabilité temporaire ( $24 \mathrm{~h}$ environ) qui permet l'absorption de protéines alimentaires intactes (Bush et Staley, 1980 ; Levieux, 1984).

Ces phénomènes, qui paraissent s'opposer à la protéolyse des protéines alimentaires dans le tractus digestif, devraient limiter l'afflux d'acides aminés alimentaires dans le sang. Or, des quantités importantes de ceux-ci apparaissent sous forme libre dans le sang périphérique chez l'agneau nouveau-né, nourri au colostrum (Patureau-Mirand et al., 1985a). Pour tenter de quantifier cet apport d'acides aminés consécutif à l'ingestion de protéines, des agneaux nouveau-nés ont été nourris avec des protéines de lait marquées par de la méthionine- ${ }^{35} \mathrm{~S}$ de façon à déterminer les flux de méthionine alimentaire dans l'intestin et dans le sang.

Ce travail a été complété par une estimation de l'activité de la transsulfuration et de celle du catabolisme de la méthionine qui sont peu connues chez le nouveau-né mais pourraient être des facteurs limitants de la fourniture de cystéine chez les prématurés (Gaull et al., 1977).

\section{Matériel et méthodes.}

Animaux et alimentation. - Quatre agneaux mâles, pesant entre 2,3 et 3,9 kg, sont séparés de leur mère dès leur naissance. Ils sont aussitôt séchés et pesés. Un cathéter est implanté, sous anesthésie locale, dans une veine jugulaire externe, pour effectuer des prises de sang sans perturber les agneaux qui sont placés dans des cages à métabolisme situées dans une pièce climatisée $119-21{ }^{\circ} \mathrm{C}$ et $50-60 \%$ d'humidité relative).

Le lait marqué a été obtenu au cours d'une expérience préalable (Champredon et al., 1986) par perfusion en continu pendant $8 \mathrm{~h}$ de 1,24 et $1,28 \mathrm{MBq} / \mathrm{h} / \mathrm{kg}$ de L-méthionine- ${ }^{35} \mathrm{~S}$ (Amersham) dans une veine jugulaire à 2 chèvres en début de lactation $\left(14^{\mathrm{e}}\right.$ et $15^{\mathrm{e}}$ jours $)$.

Les quatre agneaux reçoivent au biberon, toutes les heures, $50 \mathrm{ml}$ de lait de chèvre marqué qui sont consommés en totalité dans tous les cas. Le premier repas est ingéré à l'âge de $1 \mathrm{~h}$.

Prélèvements et analyses. - Des prélèvements de sang jugulaire sont effectués aux âges de $1 \mathrm{~h}$ (juste avant le premier repas), 4, 6 et $8 \mathrm{~h}$. A $8 \mathrm{~h}$, les agneaux sont anesthésiés par injection intraveineuse de pentobarbital sodique. Un échantillon de sang de la veine porte (sur 3 agneaux), le foie et l'intestin grêle sont prélevés.

A chaque prélèvement, les échantillons de sang sont aussitôt recueillis dans 7 fois leur volume d'éthanol $95^{\circ}$ froid $\left(-15^{\circ} \mathrm{C}\right)$ additionné de thiodiglycol $(2 \%)$ pour prévenir l'oxydation de la méthionine. A l'abattage, le foie et l'intestin grêle sont rapidement rincés avec une solution de $\mathrm{NaCl} 0,9 \%$ froid $\left(4^{\circ} \mathrm{C}\right)$, pesés, congelés et lyophilisés. Le reste du corps des agneaux est aussi rapidement congelé puis broyé de façon à préparer un échantillon homogène. 
Les teneurs en acides aminés libres du sang total sont déterminées après extraction à l'éthanol (Pawlak et Pion, 1968). Les teneurs en méthionine et cystéine des protéines du lait, des contenus digestifs, du foie, de la paroi intestinale et du reste du corps sont mesurées après une oxydation performique suivie d'une hydrolyse acide $(\mathrm{HCl} 5,5 \mathrm{~N})$ de $24 \mathrm{~h}$ selon les méthodes décrites par Prugnaud et Pion (1976). La radioactivité des échantillons est mesurée à l'aide d'un compteur à scintillation liquide (460 CD, Packard). Celle de la méthionine ou de la cystéine est déterminée sur les fractions d'effluents correspondant à ces composés, après séparation par chromatographie sur la colonne de résine échangeuse d'ions d'un analyseur (Carlo Erba).

Calcul des flux. - Les quantités de méthionine et de cystéine entrant dans l'intestin sont calculées par différence entre les quantités ingérées et celles retrouvées dans la caillette, en supposant qu'il n'y a pas d'absorption, de dégradation ou de sécrétion notables de ces composés dans la lumière du tractus digestif avant le pylore.

Selon I'équation de Stewart-Hamilton (Shipley et Clark, 1972), I'apport net ou la perte irréversible d'un composé au niveau d'un compartiment donné (souvent appelé flux), peut être calculé par le rapport entre la quantité de marqueur exogène pénétrant dans ce compartiment et son activité spécifique à ce niveau. Grâce à ce calcul, il est possible de tester la validité des 2 hypothèses suivantes :

1 - la quantité de méthionine libre collectée par le sang de la veine porte est égale à la quantité de méthionine entrée dans l'intestin. II suffit de comparer la quantité de méthionine entrée dans l'intestin ( $\mathrm{Mi}$ ) au fiux de méthionine libre au niveau de la veine porte (Fp), en reprenant I'hypothèse ci-dessus pour la méthionine radioactive, c'est-à-dire en supposant que la totalité de la radioactivité entrée dans l'intestin sous forme de méthionine- ${ }^{35} \mathrm{~S}$ passe dans le sang porte. On a alors $\mathrm{Fp}=\mathrm{Si}$. Mi/Svp où : Si est la r.a.s. de la méthionine entrant dans l'intestin, calculée par le rapport de la radioactivité de la méthionine- ${ }^{35} \mathrm{~S}$ entrant dans I'intestin à la quantité totale de méthionine arrivant à ce niveau (Mi) ; Svp est la r.a.s. de la méthionine libre du sang de la veine porte ;

2 - la quantité totale de méthionine libre, apportée par l'aliment au niveau du sang périphérique, est égale à la quantité de méthionine entrée dans l'intestin. Dans ce cas, il faut comparer la valeur du flux de méthionine au niveau du sang périphérique ( $\mathrm{Fj}$ ) calculée en faisant I'hypothèse ci-dessus pour la méthionine radioactive ( $\mathrm{Fj}=\mathrm{Si}$. Mi $/ \mathrm{Svj}$ ) à l'estimation $(\mathrm{Ft})$ du même flux, appréciée par la somme de ses composantes (flux de méthionine incorporée dans les protéines corporelles : Fs, et flux de dégradation du marqueur : Fcl. En effet, 2 expériences préalables ont montré que les estimations du flux (Fj et $F t$ ) calculées respectivement soit à partir du rapport de la radioactivité perfusée directement dans le sang jugulaire ( $r$ ) à la r.a.s. plasmatique du marqueur dans le sang périphérique (Sp), soit à partir de la somme des flux de synthèse des protéines (Fs) et de catabolisme du marqueur (FC), sont très voisines (Patureau-Mirand et al., 1985b ; Patureau-Mirand, Debras et Prugnaud, 1986). On a alors : $\mathrm{Ft}=\mathrm{r} / \mathrm{Sp} \approx \mathrm{Fs}+\mathrm{Fc}$ 
$=\mathrm{Fs}+\mathrm{Ft} . \mathrm{C}$ où $\mathrm{C}$ est le taux de catabolisme de la méthionine. On en déduit : $\mathrm{Ft}=\mathrm{Fs} /(1-\mathrm{C})$ et $\mathrm{Fc}=\mathrm{Fs} . \mathrm{C} /(1-\mathrm{C})$. Le flux de méthionine pour la synthèse des protéines (Fs) est donné par le produit de la teneur en méthionine des protéines tissulaires et de leur taux de renouvellement (Ks) estimé à l'aide de la relation simplifiée rapportée par Waterlow, Garlick et Millward (1978): Ks $=1 / \mathrm{t} . \log (\mathrm{Sp} /$ ( $\mathrm{Sp}-\mathrm{Sb})$ ), où Ks est exprimé en pour cent par heure, $t$ est l'intervalle de temps (h) qui sépare le premier repas de l'abattage, $\mathrm{Sp}$ et $\mathrm{Sb}$ sont respectivement les r.a.s. de la méthionine libre dans le sang et de la méthionine incorporée dans les protéines. Le taux de catabolisme de la méthionine (C) est le pourcentage de la méthionine radioactive entrée dans l'intestin, non retrouvée dans l'ensemble du corps.

Calculs statistiques. - Les teneurs en acides aminés libres du sang correspondant aux différents prélèvements sont comparées 2 à 2, appariées par agneau, à l'aide du test $t$ de Student de comparaison de 2 échantillons appariés.

\section{Résultats et discussion.}

Teneurs en acides aminés libres du sang. - Les teneurs en la plupart des acides aminés indispensables libres du sang (valine, isoleucine, leucine, phénylalanine, histidine) augmentent entre $1 \mathrm{~h}$ et $8 \mathrm{~h}(\mathrm{P}<0,05)$. En revanche, celles de plusieurs acides aminés non indispensables (alanine, sérine, glycine, citrulline et 3-méthylhistidine) baissent $(P<0,05)$. Cet accroissement des teneurs en acides aminés indispensables libres dans le sang périphérique, consécutif à l'ingestion de lait, est similaire à celui décrit après ingestion de colostrum de vache (PatureauMirand et al., 1985a), bien que la quantité d'acides aminés ingérée soit 4 fois plus faible $(0,51$ au lieu de $2,1 \mathrm{~g} / \mathrm{h} / \mathrm{kg})$. Ce phénomène, associé à une diminution des teneurs en plusieurs acides aminés non indispensables, suggère qu'il y a un afflux important d'acides aminés dans le sang provenant de la digestion. II peut aussi résulter d'une utilisation incomplète de ces composés pour la protéosynthèse, éventuellement par carence énergétique.

Dans le sang de la veine porte, les concentrations en acides aminés libres indispensables ou non sont plus élevées d'environ $30 \%$ que dans le sang de la veine jugulaire prélevé au même moment. Cela doit correspondre à la fourniture d'acides aminés libres au sang de la veine porte par le tractus digestif à la suite de la digestion de l'aliment, comme cela a été montré chez des animaux plus âgés : rat (Bourdel et al., 1981), porc (Rérat, 1985), mouton (Bergman et Heitman, 1980), chèvre (Greppi et Champredon, 1985).

\section{Aspects dynamiques du métabolisme de la méthionine.}

Au niveau des digesta (tabl. 1). - Dans le lait, la r.a.s. de la méthionine est 7 fois plus élevée que celle de la cystéine.

Dans les contenus de caillette, la r.a.s. de la méthionine est voisine de celle du lait ; cela signifie, soit que l'apport de méthionine par des protéines endogè- 
nes, non marquées est peu important à ce niveau, soit que la méthionine des protéines endogènes a une activité spécifique proche de celle du lait. En revanche, la r.a.s. de la cystéine est nettement plus élevée dans les contenus de caillette que dans le lait. Cela peut s'expliquer soit par des différences de r.a.s. entre la cystéine des caséines (retenues dans la caillette) et celle des protéines du lactosérum (rapidement évacuées), soit par une synthèse de cystéine à haute activité spécifique à partir de la méthionine de l'aliment.

\section{TABLEAU 1}

Méthionine et cystéine des protéines de l'aliment et des digesta (moyenne \pm écart type de l'échantillon).

\begin{tabular}{|c|c|c|}
\hline & Méthionine & Cystéine \\
\hline \multicolumn{3}{|l|}{ Radioactivité spécifique ( $\mathrm{Bq} / \mu$ mole $)$} \\
\hline Lait & 8843 & 1162 \\
\hline Contenu de caillette & $8468 \pm 1082$ & $1949 \pm 286$ \\
\hline Contenu d'intestin grêle & $2088 \pm 1182$ & $361 \pm 207$ \\
\hline Quantités ingérées ( $\mu$ moles $/ \mathrm{h} / \mathrm{kg}$ ) & $86,8 \pm 17,2$ & $46,1 \pm 9,2$ \\
\hline $\begin{array}{l}\text { Quantités entrées dans l'intestin } \\
(\mu \mathrm{moles} / \mathrm{h} / \mathrm{kg}) \ldots \ldots \ldots \ldots \ldots \ldots\end{array}$ & $41,1+5,8$ & $301+49$ \\
\hline Quantités apparemment absorbées $(*)$ & & \\
\hline$(\mu \mathrm{moles} / \mathrm{h} / \mathrm{kg}) \ldots \ldots \ldots \ldots \ldots \ldots \ldots$ & $35,5 \pm 8,0$ & $23,7 \pm 7,0$ \\
\hline Coefficient d'absorption $(\%)\left({ }^{* *}\right) \ldots$ & $97,0 \pm 0,9$ & $85,3 \pm 6,9$ \\
\hline
\end{tabular}

${ }^{*}{ }^{*}$ Méthionine ou cystéine ingérée - méthionine ou cystéine retrouvée dans les contenus de caillette et d'intestin grêle.

$\left.{ }^{* *}\right)$ Pourcentage de la quantité de radioactivité des acides aminés ${ }^{35} \mathrm{~S}$ entrée dans l'intestin, non retrouvée dans le contenu intestinal.

Dans l'intestin grêle, les r.a.s. de la méthionine et de la cystéine sont respectivement 4 et 5,4 fois plus faibles que dans la caillette. Cette baisse peut être attribuée à une importante dilution de ces acides aminés marqués par de la méthionine et de la cystéine non marquées d'origine endogène. Dans le cas de la cystéine, cela peut aussi résulter d'une différence de r.a.s. entre celle de la cystéine de la caséine et celle des protéines du lactosérum.

Compte tenu de la sécrétion non négligeable (semble-t-il) d'acides aminés soufrés d'origine endogène, nous avons retenu comme estimation des quantités de ces composés susceptibles d'être fournis à l'organisme, les quantités entrées dans l'intestin, c'est-à-dire les quantités ingérées moins les quantités retenues dans la caillette ; elles représentent 47,3 et $65,3 \%$ des quantités de méthionine et de cystéine ingérées, respectivement.

Flux de méthionine (tabl. 2). - Des quantités importantes de méthionine- ${ }^{35} \mathrm{~S}$ libre sont retrouvées au niveau du sang après ingestion du lait marqué. Dans le sang de la veine jugulaire, sa r.a.s. atteint une valeur maximale constante aux prélèvements effectués 5 et $7 \mathrm{~h}$ après le premier repas $(4734 \pm 548$ et $4716 \pm 541$ $\mathrm{Bq} / \mu$ mole, respectivement); dès $3 \mathrm{~h}$, elle est déjà égale à $88 \%$ de cette r.a.s. maximale. La r.a.s. de la méthionine- ${ }^{35} \mathrm{~S}$ libre du sang de la veine porte est significativement supérieure à celle du sang périphérique et proche de $75 \%$ de celle de 


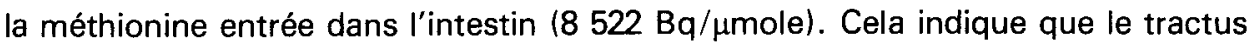
digestif libère des quantités importantes de méthionine- ${ }^{35} \mathrm{~S}$ libre dans le sang porte. Une estimation peut en être faite (voir Calcul des flux dans Matériel et méthodes) si on suppose que la totalité de la méthionine- ${ }^{35} \mathrm{~S}$ entrée dans I'intestin passe sous forme libre dans le sang de la veine porte. Cette hypothèse est plausible : d'une part, la quantité de méthionine ${ }^{-35} \mathrm{~S}$ retrouvée dans la paroi de l'ensemble de l'intestin grêle et provenant certes de l'aliment mais aussi du sang artériel, est inférieure ou égale à $11 \%$ de la quantité entrée dans l'intestin ; d'autre part, la méthionine paraît être peu catabolisée dans la paroi intestinale (Salter et al., 1985 ; voir aussi le paragraphe ci-dessous). Cette estimation de l'apport net de méthionine libre au niveau de la veine porte $(\mathrm{Fp}=53,0 \pm 6,5 \mu \mathrm{moles}(\mathrm{h} / \mathrm{kg})$ est supérieure à la quantité de méthionine entrée dans l'intestin. Cela indique que l'hypothèse selon laquelle la totalité de la méthionine entrée dans l'intestin pénètre sous forme libre dans le sang porte, ne peut être retenue ; une certaine fraction, soit ne parvient pas dans le sang de la veine porte (n'est pas absorbée ou est incorporée dans les protéines intestinales ou ne prend pas la voie sanguine), soit pénètre dans le sang de la veine porte sous forme de protéines ou de peptides. L'incorporation de méthionine dans les protéines intestinales ne peut rendre compte d'une telle différence. L'hypothèse d'un défaut d'absorption de la méthionine est en grande partie invalidée par le fait que seulement $3 \%$ de la méthionine${ }^{35} \mathrm{~S}$ entrée dans l'intestin est retrouvée dans le contenu intestinal (tabl. 1) et $q^{\prime}$ 'une fraction a une origine endogène.

Le calcul du taux de synthèse des protéines dans le corps entier $(8,7 \pm 0,3 \% / j)$ à partir de la r.a.s. de la méthionine des protéines tissulaires

TABLEAU 2

Radioactivités spécifiques de la méthionine libre du sang et de la méthionine des protéines tissulaires. Flux de méthionine.

\begin{tabular}{|c|c|c|c|c|c|c|}
\hline $\begin{array}{l}\text { Numéros des agneaux } \\
\text { Poids }(\mathrm{kg})\end{array}$ & $\begin{array}{l}43 \\
3,92\end{array}$ & $\begin{array}{l}44 \\
2,79\end{array}$ & $\begin{array}{l}45 \\
2,34\end{array}$ & $\begin{array}{l}46 \\
3,39\end{array}$ & $\begin{array}{c}\text { Moyenne } \\
3,11\end{array}$ & $\begin{array}{c}\text { Ecart type } \\
0,69\end{array}$ \\
\hline \multicolumn{6}{|c|}{$\begin{array}{l}\text { Radioactivité spécifique de la } \\
\text { méthionine libre du sang }(\mathrm{Bq} / \mu \text { mole })\end{array}$} & $\begin{array}{l}542 \\
368\end{array}$ \\
\hline $\begin{array}{l}\text { Radioactivité spécifique de la } \\
\text { méthionine des protéines }(\mathrm{Bq} / \mu \\
\text { foie } \\
\text { intestin grêle } \\
\text { corps entier }\end{array}$ & $\begin{array}{r}\text { mole) } \\
435 \\
363 \\
112\end{array}$ & $\begin{array}{l}385 \\
657 \\
131\end{array}$ & $\begin{array}{l}400 \\
739 \\
110\end{array}$ & $\begin{array}{l}455 \\
700 \\
125\end{array}$ & $\begin{array}{l}419 \\
615 \\
120\end{array}$ & $\begin{array}{r}32 \\
171 \\
10\end{array}$ \\
\hline \multicolumn{7}{|l|}{ Flux de méthionine $(\mu$ moles $/ \mathrm{h} / \mathrm{kg}$ ) } \\
\hline $\begin{array}{l}\text { synthèse protéique (Fs) } \\
\text { catabolisme (Fc) } \\
\text { total }(\mathrm{Ft})\end{array}$ & $\begin{array}{r}63,6 \\
5,2 \\
68,8\end{array}$ & $\begin{array}{r}72,5 \\
7,0 \\
79,5\end{array}$ & $\begin{array}{l}69,9 \\
16,9 \\
86,8\end{array}$ & $\begin{array}{r}60,9 \\
0,3 \\
61,2\end{array}$ & $\begin{array}{r}66,7 \\
6,6 \\
73,3\end{array}$ & $\begin{array}{r}5,3 \\
7,4 \\
11,9\end{array}$ \\
\hline $\begin{array}{l}\text { dans le sang périphérique }(\mathrm{Fi}) \\
\text { dans le sang porte }(\mathrm{Fp}) \\
\text { dans l'intestin grêle (Mi) }\end{array}$ & $\begin{array}{l}67,5 \\
52,2 \\
36,6\end{array}$ & $\begin{array}{l}76,1 \\
59,8 \\
43,3\end{array}$ & $\begin{array}{l}93,3 \\
\mathrm{NM} \\
48,2\end{array}$ & $\begin{array}{l}61,2 \\
46,9 \\
36,1\end{array}$ & $\begin{array}{l}74,5 \\
53,0 \\
.41,1\end{array}$ & $\begin{array}{r}14,0 \\
6,5 \\
5,8\end{array}$ \\
\hline
\end{tabular}

NM : non mesuré.

* Ecart type de l'échantillon. 
(tabl. 2) et de la r.a.s. de la méthionine libre plasmatique permet de connaître le flux de méthionine sanguine utilisée pour la protéosynthèse : $F s=66,7 \pm 5,3 \mu$ moles/ $\mathrm{h} / \mathrm{kg}$. Connaissant la quantité de méthionine catabolisée $(6,6 \pm 7,4 \mu$ moles $\mathrm{h} / \mathrm{kg}$ très variable selon les agneaux, voir ci-dessous), le flux total $(\mathrm{Ft})$ de méthionine peut être évalué à $73,3 \mu \mathrm{moles} / \mathrm{h} / \mathrm{kg}$. Cette valeur est proche de l'estimation obtenue en supposant que la totalité de la méthionine entrée dans l'intestin pénètre sous forme libre dans le compartiment du sang périphérique ( $\mathrm{Fj}=74,5 \mu \mathrm{moles} / \mathrm{h} / \mathrm{kg}$ ). II semble donc que la quasi-totalité de la méthionine entrée dans l'intestin a été mise à la disposition de l'organisme sous forme libre dans le sang périphérique. Cette estimation est supérieure aux $73 \%$ trouvés précédemment par une autre méthode (Patureau-Mirand et al., 1985a) chez des agneaux nouveau-nés alimentés avec du colostrum de vache. Cette différence peut tenir à la nature des protéines ingérées qui, dans le cas du colostrum, sont en grande partie représentées par des immunoglobulines, celles-ci étant retrouvées en partie intactes dans la circulation sanguine.

Le rapprochement des observations effectuées au niveau du sang de la veine porte et du sang périphérique confirme l'hypothèse que l'augmentation des teneurs en acides aminés indispensables libres du sang après l'ingestion de lait résulte en grande partie de l'afflux d'acides aminés alimentaires.

Catabolisme de la méthionine et métabolisme de la cystéine. - La quantité de méthionine catabolisée est très différente chez les 4 agneaux ainsi qu'en témoignent les valeurs du bilan de la méthionine, apprécié par la différence des radioactivités de la méthionine entrée dans l'intestin et de celle retrouvée dans l'organisme (fig. 1). Les bilans de cystéine montrent que, chez 3 agneaux, il y a une synthèse apparente de cystéine, d'autant plus importante que le taux de catabolisme de la méthionine est élevé. L'absence de synthèse apparente de cystéine est observée chez l'agneau 46 qui dégrade les plus faibles quantités de méthionine. L'augmentation parallèle de la r.a.s. de la cystéine des protéines du foie en fonction du taux de catabolisme de la méthionine, suggère une transsulfuration active dans cet organe chez l'agneau nouveau-né. En revanche, cette voie métabolique

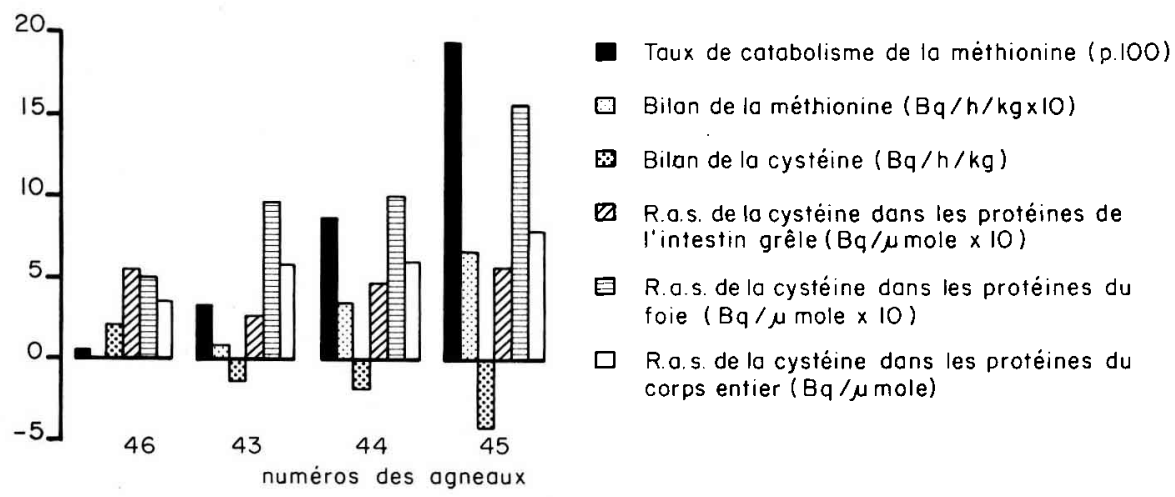

FIG. 1. - Métabolisme des acides aminés soufrés. 
serait peu active dans l'intestin grêle car le rapport des r.a.s. de la cystéine à celles de la méthionine (de 0,072 à 0,078 selon les agneaux) est comparable à celui mesuré dans les protéines de lait entrées dans l'intestin et nettement inférieur à celui mesuré dans le foie. Ce dernier varie de $0,11 \mathrm{chez}$ l'agneau qui catabolise peu la méthionine à 0,39 chez celui qui la catabolise le plus.

L'activité de la transsulfuration dans le foie paraît donc être un des principaux facteurs du catabolisme de la méthionine chez le nouveau-né comme chez le mouton plus âgé (Pisulewski et Buttery, 1985). Cette activité qui paraît très variable pourrait être insuffisante pour couvrir les besoins en cystéine de certains agneaux nouveau-nés qui, comme l'agneau 46 , catabolisant peu la méthionine, ne peuvent bénéficier d'une synthèse nette de cystéine.

\section{Conclusion.}

La digestion des protéines de lait par l'agneau nouveau-né paraît relativement intense dans le tractus digestif, dès les premières heures après la naissance. II semble qu'elle puisse être complétée par une hydrolyse ultérieure des protéines alimentaires absorbées intactes. La transsulfuration qui est active dès la naissance chez l'agneau nouveau-né a cependant une intensité très variable selon les individus.

Reçu en août 1986.

Accepté en février 1987.

Remerciements. - Nous tenons à remercier P. Limouzin de son amicale collaboration.

\section{Références}

BERGMAN E. N., HEITMAN R. N., 1980. Integration of whole-body amino acid metabolism, 69-84. In BUTTERY P. J., LINDSAY D. B., Protein deposition in animals, Butterworths, Londres.

BOURDEL G., KANDE J., ROBIN D., ROBIN P., 1981. Quantitative and qualitative circadian variations of amino acid intestinal efflux in mixed-fed and in protein-meal-fed-rats. J. Nutr., 111. 1528-1535.

BUSH L. J., STALEY T. E., 1980. Absorption of colostral immunoglobulins in newborn calves. J. Dairy Sci., 63, 672-680.

CHAMPREDON C., DEBRAS E., PION R., ARNAL M., 1986. Influence du début de lactation sur les flux de méthionine chez la chèvre. Résultats préliminaires. Reprod. Nutr. Dévelop., 26, $341-342$.

GAULL G. E., RASSIN D. K., RAIHA N. C. R., HEINHNEN K., 1977. Milk protein quantity and quality in low-birth-weight infants. J. Pediatr., 90, 348-355.

GREPPI G. F., CHAMPREDON C., 1985. Studio del l'absorbimento intestinale di azoto aminoacidico nella capra. VI. Congr. naz. Assoc. sci. Perugia, S. Pietro, Italie.

GUILLOTEAU P., CORRING T., TOULLEC R., VILLETTE-HOUSSIN Y., ROBELIN J., 1985. Abomasum and pancreas enzymes in the newborn ruminant : effect of species, breed, sex and weight. Nutr. Report. int., 6, 1231-1236.

LEVIEUX D., 1984. Transmission de l'immunité passive colostrale : le point des connaissances, 345369. In JARRIGE R., Physiologie et pathologie perinatales chez les animaux de ferme, I.N.R.A., Paris. 
PATUREAU-MIRAND P., BERNARD O., PRUGNAUD J., LEVIEUX D., PION R., 1985a. Métabolisme protéique de l'agneau nouveau-né. - I. Conséquences de l'ingestion de colostrum sur la glycémie, la gammaglobulinémie et les teneurs en acides aminés libres des tissus. Reprod. Nutr. Dévelop., 25, 1047-1060.

PATUREAU-MIRAND P., BERNARD O., PRUGNAUD J., ARNAL M., 1985b. Métabolisme protéique de I'agneau nouveau-né. - II. Influence de l'alimentation sur les flux et le taux de renouvellement des protéines. Reprod. Nutr. Dévelop., 25, 1061-1073.

PATUREAU-MIRAND P., DEBRAS E., PRUGNAUD J., 1986. Influence de l'ingestion de lactose sur le métabolisme protéique de l'agneau nouveau-né. Reprod. Nutr. Dévelop., 26, 677-682.

PAWLAK M., PION R., 1968. Influence de la supplémentation des protéines du blé par des doses croissantes de lysine sur la teneur en acides aminés libres du sang et du muscle du rat en croissance. Ann. Biol. anim. Bioch. Biophys., 8, 517-530.

PISULEWSKI P. M., BUTTERY P. J., 1985. The effect of increasing methionine supply on the methionine conversion to cyst(e)ine in sheep. Br. J. Nutr., 54, 121-129.

PRUGNAUD J., PION R., 1976. Dosage des acides aminés dans les aliments. In Journées de Biochimie: l'analyse des acides aminés. Beckman, Paris, $23 \mathrm{p}$.

RÉRAT A., 1985. Intestinal absorption of end products from digestion of carbohydrates and proteins in the pig. Arch. Tierernähr., 35, 461-480.

SALTER D. N., SMITH R. H., MONTGOMERY A. 1., HUDSON A., QUELCH D., 1985. Gut metabolism of amino acids during absorption. Proc. XIIIth Int. Congr. Nutr., Brigthon, p. 94.

SHIPLEY R. A., CLARK R. E., 1972. Tracer methods for in vivo kinetics. Theory and applications, 145-162, Acad. Press, New York.

WATERLOW J. C., GARLICK P. J., MILLWARD D. J., 1978. Protein turnover in mammalian tissues and in the whole body. North Holland, Amsterdam, $804 \mathrm{p}$. 